\title{
Decisional Conflict and User Acceptance of Multicriteria Decision-Making Aids*
}

\author{
Jeffrey E. Kottemann and Fred D. Davis \\ Graduate School of Business Administration, The University of Michigan, \\ Ann Arbor, MI 48109-1234
}

\begin{abstract}
Despite the development of increasingly sophisticated and refined multicriteria decision-making (MCDM) methods, an examination of the experimental evidence indicates that users most often prefer relatively unsophisticated methods. In this paper, we synthesize theories and empirical findings from the psychology of judgment and choice to provide a new theoretical explanation for such user preferences. Our argument centers on the assertion that the MCDM method preferred by decision makers is a function of the degree to which the method tends to introduce decisional conflict. The model we develop relates response mode, decision strategy, and the salience of decisional conflict to user preferences among decision aids. We then show that the model is consistent with empirical results in MCDM studies. Next, the role of decisional conflict in problem formulation aids is briefly discussed. Finally, we outline future research needed to thoroughly test the theoretical mechanisms we have proposed.

Subject Areas: Decision Analysis, Decision Processes, Decision Support Systems, and Human Information Processing.
\end{abstract}

\section{INTRODUCTION}

The development of multicriteria decision-making (MCDM) aids has been motivated both by the increasing recognition of the multicriteria nature of managerial decision tasks and by the increasing power and accessibility of computers [16]. Despite the growth and refinement in MCDM methods, they have not been widely adopted [22]. An examination of experimental studies (e.g., [8], [9], [22], and [32]) reveals that users, given a choice among decision aids, most often prefer relatively unsophisticated methods. Indeed, unaided conditions may be preferred over any formal method. For example, Narasimhan and Vickery [22] found that the mean satisfaction score for ad hoc solutions initially generated by decision makers was higher than for solutions generated after use of MCDM aids.

In this paper, we synthesize theories and empirical findings from the psychology of judgment and choice to provide a new theoretical explanation for such user preferences. Our argument centers on the assertion that the MCDM method preferred by decision makers is a function of the degree to which the method tends to introduce decisional conflict. The key mechanisms we propose are (1) the effect of response mode on decision-making strategy, (2) the effect of decision-making strategy on the salience of decisional conflict, and (3) the effect of decisional conflict on decision-maker attitudes. Figure 1 illustrates these mechanisms. In the following section we begin by explaining the latter two mechanisms and then integrate them with the first.

*The comments of Michael Cohen, Dan Dolk, the Associate Editor, and two referees, and the support of the Division of Research, Graduate School of Business Administration, The University of Michigan, are gratefully acknowledged. 
Figure 1: The decisional conflict model.

\section{The Model}

Response mode required (and, in the case of choice mode, composition of the choice set)<smiles></smiles>

Decision strategy used<smiles></smiles>

Salience of decisional conflict<smiles></smiles>

Attitudes toward decision made and aid
Explanation

\author{
Matching engenders use of \\ compensatory strategies. Choice \\ among many alternatives engen- \\ ders use of noncompensatory \\ strategies.
}

Compensatory strategies highlight trade-off conflicts while noncompensatory strategies do not.

Highlighting decisional conflict negatively affects attitudes.

\section{PSYCHOLOGY OF JUDGMENT AND CHOICE}

When presented with a judgment or choice task, people can and do employ a number of different strategies. These strategies can be classified as noncompensatory and compensatory (for a review, see [1]). Noncompensatory strategies, such as a lexicographic or elimination-by-aspects rules, do not involve assessing attribute trade-offs among alternatives. For example, if a person uses a strategy that eliminates all apartments with less than two bedrooms, then this noncompensatory strategy avoids explicitly trading off the number of bedrooms with other criteria such as rent and distance to work. On the other hand, compensatory strategies involve explicit assessments of trade-offs. For example, a person might explicitly determine that he/she is willing to trade-off one bedroom for a $\$ 200$ reduction in rent. In effect, decision strategies determine the degree to which decisional conflict is made salient. In particular, compensatory strategies highlight trade-off conflicts while noncompensatory strategies do not.

What is the effect of strategy-induced conflict on a decision maker's attitudes? With use of a noncompensatory strategy the chosen alternative may be considered unequivocally best by the decision maker simply because the chosen alternative was superior on the subset of attributes that were assessed. In this case, decisional conflict is masked, and one would expect a decision maker to be quite confident in his or her decision. With a compensatory strategy, on the other hand, trade-offs are explicitly considered and an alternative is chosen with the knowledge that other alternatives are better along some dimensions. Compensatory strategies are "conflict confronting," whereas noncompensatory strategies allow evaluation to proceed without facing the computational and emotional difficulties of making trade-offs [13]. Trade-off conflict is a major source of decisional stress [19]. (For a review of conflict in group decision making, see [27].)

Zakay [34] empirically demonstrated the effect of decision strategy on confidence (also see [4] [14]). In his experiment, experienced emergency room nurses were given patient status profiles and asked to make decisions on which of two 
patients the nurses would attend to first. Zakay [34] found that decisions made using a noncompensatory strategy were associated with significantly higher levels of post-decisional confidence than when a compensatory strategy was used. Similar results were obtained in studies by Adelbratt and Montgomery [3] and by Wright [33]. These results suggest that the higher trade-off conflict salience induced through use of a compensatory strategy results in lower post-decisional confidence.'

What leads decision makers to adopt compensatory and noncompensatory strategies? One important factor is the type of response mode required of the decision maker (see [7] [31]). Take, for example, a choice versus a matching mode. With a choice mode, the decision maker is presented with two or more alternatives and asked to choose between them. With a matching mode, the decision maker is presented with two alternatives, but one attribute of one alternative is left as a free variable and the decision maker is asked to supply a value for the free variable that makes him/her indifferent between the two alternatives. ${ }^{2}$ As Tversky, Sattath, and Slovic [31] discussed in detail, decision makers ate more likely to use compensatory strategies for matching tasks than for choice tasks.

For example, consider the following matching question:

$\begin{array}{cc}\text { RENT } & \text { BEDROOMS } \\ \$ 500 & 2 \\ X ? & 3\end{array}$

The decision maker is being asked how much more she is willing to pay for three bedrooms than two bedrooms-an explicit trade-off judgment. On the other hand, the same situation presented as a choice (e.g., with $\$ 800$ substituted for $X$ ) encourages neither a compensatory nor a noncompensatory strategy. The decision maker is free to use any strategy she wishes.

With a choice response mode, another important factor is the composition of the choice set. In a choice mode, the use of noncompensatory strategies is more likely as the number of alternatives increases [1] [23]. This generalization appears to hold for both risky and riskless choice problems [1]. Therefore, when decision makers are asked to choose from among an increasing number of alternatives, they tend to adopt simple, noncompensatory strategies in order to deal with the greater problem complexity and, through the use of such strategies, decisional conflict is avoided.

Taken together, the above analysis suggests that the type of response required of a decision maker - and, in the case of choice, the size of the choice set-influences the type of cognitive decision strategy used. This, in turn, influences users' confidence and acceptance of decision aids by affecting the salience of decisional conflict (Figure 1). In the following section we examine MCDM laboratory studies from this point of view to assess the role of response mode, decision strategy, and

\footnotetext{
${ }^{1}$ The issue of whether confidence is well calibrated to actual decision-making performance, while of vital interest, is beyond the scope of this note (see, e.g., [14]).

${ }^{2}$ For example, given a two bedroom apartment that rents for $\$ 500$ per month, what would the rent for a three bedroom apartment have to be to make you indifferent between the two apartments?
} 
resulting trade-off conflict, as possible mechanisms underlying user preferences for "simpler" MCDM methods.

\section{AN ANALYSIS OF MCDM METHODS AND LABORATORY STUDIES}

Our argument is that the degree of decisional conflict affects user preference for decision aids, and that decisional conflict is determined by the design of the decision aid.

\section{MCDM Methods: Response Modes and Decisional Conflict}

There are various response modes employed by MCDM methods. Each has implications regarding the decisional conflict experienced by decision makers. Below we list the types of MCDM response modes that have been subject to empirical study, ordered by decreasing potential for decisional conflict.

1. High potential for decisional conflict. The majority of MCDM methods require decision makers to make explicit trade-off judgments. Perhaps the most demanding is the method of Geoffrion, Dyer, and Feinberg [15] which requires that decision makers specify their marginal rates of substitution between criteria. At each cycle with this method, the decision maker is expected to provide an estimate of his/her marginal rates of substitution between the criteria and resolution of the gradient step-size. Other methods that require explicit trade-off judgments are Benayoun, De Montgolfier, Tergny, and Laritchev [6], Haimes and Hall [17], Saaty [26], and Zionts and Wallenius [37].

2. Medium potential for decisional conflict. Methods such as Posner and Wu's [24] allow decision makers to specify an ideal, but potentially infeasible solution. This method then finds the closest feasible solution and has decision makers specify a new ideal solution. If the ideal solution specified by the decision maker creates an (inflated) aspiration level (as Brockhoff [8] has suggested), then interaction with the MCDM aid may cause decisional conflict by making salient the disparity between desires and feasibility (and may lead the decision maker to make trade-offs to attain feasibility).

3. Low potential for decisional conflict. In contrast to the above, methods such as Steuer and Choo's [29] do not make decisional conflict explicit. Steuer and Choo's method has the decision aid automatically generate six efficient solutions from which a decision maker chooses his or her most preferred solution. The discussion in the previous section, as represented by Figure 1, predicts that decision makers will prefer MCDM methods that leave decisional conflict implicit. In particular, it predicts that methods such as Steuer and Choo's [29] will be preferred. The results of MCDM laboratory research, while scarce, are consistent with this reasoning.

\section{Analysis of MCDM Laboratory Studies}

Buchanan and Daellenbach [9] compared MCDM methods using a production scheduling task. Four different MCDM methods were assessed in the study. Two of the methods [17] [37] require explicit trade-off judgments. The third method [24] allows decision makers to specify a desired solution in terms of the criteria; the method then finds the closest feasible, efficient solution and has decision 
makers specify a new desired solution. The fourth method [29] has the decision maker choose from MCDM-generated sets of six solutions. While the first three methods involve either explicit trade-off judgments or the generation of desired solutions followed by revision to attain feasibility, the method of Steuer and Choo [29] does not force decision makers to experience decisional conflict.

Subjects were asked to rate each method on a preference for use scale. Preference for use was lowest for the two methods that involve trade-off judgments, followed by the method of Posner and $\mathrm{Wu}$ [24] that involves revision to attain feasibility. Subjects expressed significantly higher preference for the Steuer and Choo method as well as higher confidence in the solution attained through use of the method, although the confidence difference did not reach statistical significance.

Brockhoff [8] contrasted Steuer's [28] and Benayoun, et al.'s [6] methods for aiding in the purchase of automobiles. As above, Steuer's method asks users to choose among multiple MCDM-generated solutions, whereas Benayoun's method requires users to make explicit trade-off judgments. Steuer's low-conflict method was favored by a majority of subjects. In particular, Steuer's method was rated significantly higher for instrumentality, which is correlated with the perceived usefulness of MCDM systems [8]. Brockoff's findings are consistent with those of Buchanan and Daellenbach [9] (discussed earlier) and with our proposition.

Wallenius [32] compared performance of the interactive methods of Benayoun, et al. [6], Geoffrion, et al. [15], and an unstructured method using a production scheduling task similar to that used in Buchanan and Daellenbach [9]. Subjects included both undergraduate students and managers with experience in the task domain. The first two methods require explicit trade-off judgments, while the unstructured method was essentially an automated trial-and-error procedure. While the students' preference rankings for the methods were quite random, the managers "gave an overall preference to the unstructured approach over the more sophisticated methods" [32, p. 1390]. And, while there was no statistically significant difference in confidence associated with the methods, "by analyzing the responses of the subjects it is possible to claim that the difficulty of giving the MRS [marginal rates of substitution] in the Geoffrion method weakened the confidence in its solution" [32, p. 1391].

Narasimhan and Vickery [22] compared the Zionts and Wallenius [36] method with Saaty's [26] analytical hierarchy process. Both methods involve explicit tradeoff judgments between criteria, albeit at different times in the decision-making process and by quite different trade-off elicitation approaches. Subjects' attitudinal responses after using the decision aids showed no significant difference between the two MCDM methods regarding users' satisfaction with solution ratings. What is surprising at first glance, and what could be considered "counterintuitive" [22, p. 886], is that the satisfaction with solution ratings associated with ad hoc solutions initially generated by decision makers were higher (though not significantly) than for solutions that were generated with help from the MCDM aids.

The available research on users' relative preference among MCDM techniques is consistent with our proposition that the increased salience of trade-off conflict, induced by response mode, leads to reduced confidence in, and preference for, a given technique. As the above studies indicate, methods that require explicit tradeoff judgments such as Benayoun, et al. [6], Geoffrion, et al. [15], Haimes and Hall 
[17], Saaty [26], and Zionts and Wallenius [35] [36] [37] tend to be less preferred than methods that do not force trade-off conflict to become salient such as Posner and $\mathrm{Wu}$ [24], Steuer [28], and Steuer and Choo [29]. Although the available evidence is by no means conclusive, it is suggestive of an interesting pattern that warrants future research. One interesting question is whether the phenomenon generalizes to other types of decision aids.

\section{Generalization to Problem Formulation Aids}

In a typical MCDM study, the experimental tasks presented to subjects are pre-formulated: Both criteria and constraints are predefined such that the main task of the subjects is to explore, not to formulate, a problem space. A less-structured decision task, such as "What career will you pursue?", entails more than determining a desired, feasible solution subject to a set of predefined constraints in order to maximize preference over a set of predefined criteria. Rather, the decision options, criteria, and constraints must be formulated. Thus, potential decisional conflict is not only a function of the decision strategy used to assess alternatives, but also a function of the activities of problem formulation. Specifically, trade-off conflict may increase when additional conflicting criteria are formulated. Take for example, selecting among bids. If cost is the sole criterion, then one may merely select the lowest cost bid. If during problem formulation, reliability of the bidding contractor is introduced as another criterion (and assuming the cost and reliability are conflicting), potential trade-off conflict increases.

Decision aids for problem formulation often strive to help decision makers formulate exhaustive sets of objectives and alternatives. While, prima facie, such broadening of the problem structure may be deemed desirable, it is also possible that decision makers' subjective impressions of decision quality may be negatively affected due to increases in decisional conflict. Such negative impressions may well form despite an aid's positive influence on actual decision quality. Cats-Baril and Huber [10] reported on the effects of a purpose-expansion heuristic which was used by students for career planning. Students who used the purpose-expansion heuristic formulated more objectives and alternatives. They also generated higher quality career plans as rated by career counselors. Yet, users of the heuristic expressed significantly lower confidence in their career plans.

Thus, consistent with a decisional conflict argument, Cats-Baril and Huber [10] found reduced decision confidence with the use of conflict-inducing methods. Similar results were obtained in a recent study by Abualsamh, Carlin, and McDaniel [2]. The fact that objective decision quality was actually enhanced in both cases underscores the possibility that users may avoid helpful techniques due to misperceptions of their effectiveness. (An interesting counterpoint example is provided in the case study of Humphreys and McFadden [18]. In this case, the multi-attribute utility decomposition aid, MAUD, was judged helpful to decision makers when it helped them eliminate conflicting objectives.) These results suggest that the effects of decisional conflict may come into play with a variety of decision-aiding technologies.

\section{Future Empirical Research}

While existing MCDM study results are consistent with the conflict model developed here, the only variables of our model that were measured in those 
experiments were decision maker attitudes given MCDM method (response mode) manipulations. The intermediate mechanisms we have proposed were not measured. Future research is needed to test whether these intermediate mechanisms do indeed come into play. Specifically, three propositions must be tested in an MCDM context.

1. MCDM methods (response modes) influence the decision strategies adopted by decision makers.

Here, process tracing methods could be used to test the effect of the various MCDM response mode methods on decision strategy. For example, process tracing could be used to test whether decision makers do indeed adopt noncompensatory strategies while using Steuer and Choo's [29] method.

2. Decision strategies adopted by decision makers while using MCDM aids affect the decisional conflict they experience.

Here, measures could be used to assess the effects of MCDM-engendered decision strategies on the degree of decisional conflict experienced by decision makers. These measures could be in the form of a questionnaire or physiological measures such as heart rate could be used ([19]).

3. The decisional conflict experienced while using MCDM aids affects the perceived usefulness of MCDM methods.

Given the existence of the former two mechanisms, and measures of the perceived usefulness of the MCDM aids similar to those in previous studies, the correlation of decisional conflict experienced and perceived usefulness can be ascertained.

\section{CONCLUSION}

In this note we have presented a model centered around decisional conflict which helps explain why decision makers may fail to adopt sophisticated (e.g., trade-off driven) methods for MCDM problems. We have also outlined future research needed to thoroughly test the model. One direction we have not discussed is the development of decision aids that allow decision makers the option of avoiding decisional conflict (e.g., [5]); indeed, the reduction of decisional stress has been proposed as a desirable feature for decision aids [25]. However, three practical matters must be kept in mind.

First, some degree of decisional stress is necessary to insure that decision makers are vigilant information processors [19]. Second, many real-life decision contexts require decision makers to formally justify their decisions (which was not the case in any of the MCDM studies discussed here). In such contexts, decision makers can be lead to recognize the need to confront difficult value trade-offs [30]. Last, while perceived usefulness is a major determinant in the adoption of computer-based technologies [11] [12], subjective judgments such as confidence and perceived usefulness of decision aids are not reliable predictors of actual performance effects [11] [14] [20] [21].

The analysis presented here suggests that decision technologies that increase decisional conflict may lead users to feel that their decision performance has been negatively affected, while in fact the technology improved actual performance. Research on user acceptance has found that adoption of new technologies is largely driven by users' perceptions of performance improvement, despite whatever real performance improvements there are. Together, these streams of research suggest 
the unfortunate possibility that people will avoid beneficial decision aids because of misperceptions created by heightened decision conflict. [Received: November 27, 1989. Accepted: August 21, 1990.]

\section{REFERENCES}

[1] Abelson, R. P., \& Levi, A. Decision making and decision theory. In G. Linsay \& E. Aronson (Eds.), The handbook of social psychology (3rd ed.). New York: Random House, 1985.

[2] Abualsamh, R. A., Carlin, B., \& McDaniel, R. R., Jr. Problem structuring heuristics in strategic decision making. Organizational Behavior and Human Decision Processes, 1990, 45, 159-174.

[3] Aldelbratt, T., \& Montgomery, H. Attractiveness of decision rules. Acta Psychologica, 1980, 45, 177-185.

[4] Allwood, C. M., \& Montgomery, H. Response selection strategies and realism of confidence judgments. Organizational Behavior and Human Decision Processes, 1987, 39, 365-383.

[5] Badinelli, R. D., \& Baker, J. R. Multiple attribute decision making with inexact value-function assessment. Decision Sciences, 1990, 2l(2), 318-336.

[6] Benayoun, R., De Montgolfier, J., Tergny, J., \& Laritchev, O. Linear programming with multiple objective functions: STEP-method (STEM). Mathematical Programming, 1971, I(3), 366-375.

[7] Billings, R. S., \& Scherer, L. L. The effects of response mode and importance on decision-making strategies: Judgement versus choice. Organizational Behavior and Human Decision Processes, 1988, 41, 1-19.

[8] Brockhoff, K. Experimental test of MCDM algorithms in a modular approsch. European Journal of Operations Research, 1985, 22, 159-166.

[9] Buchanan, J. T., \& Daellenbach, H. G. A comparative evaluation of interactive solution methods for multiple objective docision models. European Journal of Operations Research, 1987, 24, 353-359.

[10] Cats-Baril, W. L., \& Huber, G. P. Decision support systems for ill-structured problems: An empirical study. Decision Sciences, 1987, 18, 350-372.

[11] Davis, F. D. Perceived usefulness, perceived ease of use, and user acceptance of information technology. Management Information Systems Quarterly, 1989, 13(3), 319-339.

[12] Davis, F. D., Bagozzi, R., \& Warshaw, P. User acceptance of computer technology: A comparison of two theoretical models. Management Science, 1989, 35, 982-1003.

[13] Einhom, H. J., \& Hogarth, R. M. Behavioral decision theory: Processes of judgment and choice. Annual Review of Psychology, 1981, 32, 53-88.

[14] Fischhoff, B., Slovic, P., \& Lichtenstein, S. Knowing with certainty: The appropriateness of extreme confidence. Journal of Experimental Psychology: Human Perception and Performance, $1977,3,552-564$.

[15] Geoffrion, A. M., Dyer, J. S., \& Feinberg, A. An interactive approach for multicriterion optimization, with an application to the operation of an academic department. Management Science, 1972, 19(4), 357-368.

[16] Golden, B. L., Hevner, A., \& Power, D. Decision insight systems for microcomputers: A critical evaluation. Computers and Operations Research, 1986, 13(2/3), 287-300.

[17] Haimes, Y. Y., \& Hall, W. A. Multiobjectives in water resources systems analysis: The surrogate worth tradeoff methods. Water Resources Research, 1974, 10, 615-624.

[18] Humphreys, P., \& McFadden, W. Experiences with MAUD: Aiding decision structuring versus bootstrapping the decision maker. Acta Psychologica, 1980, 45, 51-69.

[19] Janis, I. L., \& Mann, L. Decision making: A psychological analysis of conflict, choice, and commitment. New York: The Free Press, 1977.

[20] Kahneman, D., \& Tversky, A. On the psychology of prediction. Psychological Review, 1973, $80(4), 237-251$.

[21] Kottemann, J. E., \& Remus, W. E. Evidence and principles of functional and dysfunctional DSS. International Journal of Management Science (Omega), 1987, 15(2), 135-144.

[22] Narasimhan, R., \& Vickery, S. K. An experimental evaluation of articulation of preferences in multiple criterion decision-ınaking (MCDM) methods. Decision Sciences, 1988, 19, 880-888.

[23] Payne, J. Contingent decision behavior. Psychological Bulletin, 1982, 92, 382-402.

[24] Posner, M. E., \& Wu, C. T. Linear max-min programming. Mathematical Programming, 1981, $20,166-172$.

[25] Rohrmann, B. Evaluating the usefulness of decision aids: A methodological perspective. In B. Brehmer, H. Jungermann, P. Lourens \& G. Sevon (Eds.), New directions in research on decision making. New York: North-Holland, 1986. 
[26] Saaty, T. L. The analytical hierarchy process. New York: McGraw-Hill, 1980.

[27] Schwenk, C. R. Conflict in organizational decision making: An exploratory study of its effects in for-profit and not-for-profit organizations. Management Science, 1990, 36(4), 436-448.

[28] Steuer, R. E. An interactive multiple objective linear programming procedure. In M.K. Starr \& M. Zeleny (Eds.), Multiple criteria decision making. Amsterdam: North-Holland, 1977.

[29] Stever, R. E., \& Choo, E. U. An inleractive weighted Tchebycheff procedure for multiple objective programming. Mathematical Programming, 1983, 26, 326-344.

[30] Tetlock, P. E. Accountability: The neglected social context of judgment and choice. Research in Organizational Behavior, 1985, 7, 297-332.

[31] Tversky, A., Sattath, S., \& Slovic, P. Contingent weighting in judgment and choice. Psychological Review, 1988, 95(3), 371-384.

[32] Wallenius, J. Comparative evaluation of some interactive approaches to multicriterion optimization. Management Science, 1975, 21(12), 1387-1396.

[33] Wright, P. Consumer choice strategies: Simplifying vs. optimizing. Journal of Marketing Research, 1975, 12, 60-67.

[34] Zakay, D. Post-decisional confidence and conflict experienced in a choice process. Acta Psychologica, 1985, 58, 75-80.

[35] Zionts, S. Multiple criteria mathematical programming: An overview and several approaches. In G. Fandel \& J. Spronk (Eds.), Multiple criteria decision methods and applications. Heidelberg: Springer-Verlag, 1985.

[36] Zionts, S., \& Wallenius, J. An interactive programming method for solving the multiple criteria problem. Management Science, 1976, 22, 652-663.

[37] Zionts, S., \& Wallenius, J. An interactive multiple objective linear programming method for a class of underlying nonlinear utility functions. Management Science, 1983, 29(5), 519-529.

Jeffrey E. Kottemann is Assistant Professor of Computer Information Systems in the School of Business Administration at The University of Michigan. He received his Ph.D. in management information systems from The University of Arizona and has published articles in Communications of the ACM, Decision Sciences, Information Systems. The Imternational Journal of Management Science, Journal of MIS, and MIS Quarterly. His current areas of research include assessing the impacts of computerized decision aids on performance and decision makers' perceptions of their performance, and devising tools and techniques for development of large-scale information systems and modeling systems.

Fred D. Davis is Assistant Professor of Computers and Information Systems at The University of Michigan Business School. He received his Ph.D. in management science from the Sloan School of Management, Massachusetts Institute of Technology. He has published articles in Management Science, MIS Quarterly, Journal of Experimental Social Psychology, and other journals. Dr. Davis' current research interests include user acceptance of computer technology and computers in decision making. 